\section{DOUBLE ACTION DENTAL PAIN RELIEF}

Nuromol is the only product to contain a fixed dose combination of ibuprofen and paracetamol. It is especially suitable for pain that requires stronger analgesia than can be achieved with ibuprofen or paracetamol alone.

A 2011 study published in Pain compared the efficacy and tolerability of Nuromol over 12 hours with that of an ibuprofen/codeine and a paracetamol/codeine combination using the

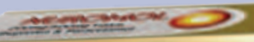

DOUBLE ACTION PAIN RELIEF dental impaction pain model.

The study concluded that one Nuromol tablet provided stronger and longer lasting pain relief than paracetamol $1,000 \mathrm{mg} /$ codeine $30 \mathrm{mg}$ (ie cocodomol), with two Nuromol tablets providing more effective pain relief than was sustained for longer than an ibuprofen $400 \mathrm{mg} /$ codeine $25.6 \mathrm{mg}$ combination.

For adult patients requiring relief of dental pain, Nuromol provides a unique, clinicallyproven treatment that provides strong pain relief that is long lasting with no ingredients known to be addictive.

For further information visit www.nuromol.co.uk.

\title{
REFINE YOUR RESTORATIVE SKILLS IN LOVELY LEUVEN
}

Learn how to create possibly the 'most beautiful composite restoration' in the historic city of Leuven, Belgium with GC.

GC G-aenial is a unique light cured composite that allows you to fabricate natural looking, aesthetic direct restorations. The hands-on G-aenial course shows you how to craft functional composite restorations; even monochromatic restorations become beautiful due to the unique chameleon effect of GC G-aenial. This unique light curable micro-ceramic composite material benefits from those all-important properties such as strength and durability, but unlike other composite materials on the market, GC G-aenial has been developed to look like natural tooth structure.

G-aenial's more natural appearance has been achieved by creating a final restoration of optimum hue, chroma and brightness, whilst minimising the paleness inherent with composites. The result is a restoration with opalescence comparable to porcelains and close to natural teeth, which would previously have been impossible to attain using traditional composite materials.

The GC G-aenial course, with ten hours of CPD, includes flights from London Heathrow or Eurostar, transfers to the hotel and training centre, one night's hotel accommodation, all meals, and all materials, models and equipment.

For full details of the

G-aenial courses, which are contained within GC's Training Calendar Booklet, or for further information contact GC UK on 01908218999.

\section{COMPLIANCE WITH MINIMUM FUSS}

Dentisan's website and new wall chart provide useful resources for practices wanting help understanding the practical implications of the HTM 01-05 guidelines. Dentisan, whose strapline is 'advanced hygiene in practice', is committed to marketing products that will provide dental practices with infection control solutions, ensuring compliance with HTM 01-05.

The recently updated wallchart features Dentisan's full range of infection control products in a neat A3 laminated poster that can be displayed in the decontamination room for easy reference. Visitors to the website can also access all the relevant compliance information and view the new Biocleanse Ultra video that compares performance against other products available on the market. Biocleanse Ultra is an alcohol free multi-surface cleaner and disinfectant that combines excellent cleaning, materials compatibility and wide spectrum disinfection, in a $\mathrm{pH}$ neutral formulation. It has proven efficacy against mycobacteria including TB, enveloped viruses such as Hepatitis B and C, HIV, bacteria, fungi and yeast.

Visit www.dentisan.co.uk. To receive a free wall chart (while stocks last) email sample@dentisan.co.uk quoting reference Chart08.

\section{A GUIDE TO CREATING BEAUTIFUL RESTORATIONS}

Dr Andreas Ender has written a comprehensive CEREC Software Guide which can easily be downloaded from www. sirona.com/en/products/digitaldentistry/cerec-chairsidesolutions/?tab=245.
'CEREC Basic information 4.0 - A Clinical Guide' is packed full of interesting and useful information with many tips and tricks to help you to get the best out of CEREC. With a step by step approach including full colour clinical shots, this guide is incredibly useful for both new and experienced users alike. For those users not yet using the v4.0 software there is a new v3.8 clinical guide available.

From the clinical principles and preparation guidelines through to finishing and glazing, this definitive guide takes you through the methods employed to create a beautiful CEREC restoration in a single appointment.

For further information contact Sirona Dental Systems on 08450715040 or email info@ sironadental.co.uk. 